\title{
Novel strategy of sirolimus plus thymalfasin and huaier granule on tumor recurrence of hepatocellular carcinoma beyond the UCSF criteria following liver transplantation: A single center experience
}

\author{
LIN ZHOU ${ }^{1,2^{*}}$, LI-CHAO PAN ${ }^{1,2^{*}}$, YONG-GEN ZHENG ${ }^{1,2^{*}}$, GUO-SHENG DU $^{1 *}$, XIAO-QIAN FU ${ }^{1}$, \\ ZHI-DONG ZHU ${ }^{1}$, JI-YONG SONG ${ }^{1}$, ZHI-JIA LIU ${ }^{1}$, XIANG-ZHENG SU ${ }^{2}$, WEN CHEN ${ }^{2}$, \\ DE-HUA ZHENG ${ }^{1}$, LONG-LONG SUO ${ }^{1}$ and SHAO-ZHEN YANG ${ }^{1}$ \\ ${ }^{1}$ Department of Hepatobiliary Surgery, Organ Transplant Institute, Chinese PLA 309th Hospital, Beijing 100091; \\ ${ }^{2}$ Department of Hepatobiliary Surgery, Chinese PLA General Hospital, Beijing 100853, P.R. China
}

Received September 24, 2017; Accepted March 15, 2018

DOI: $10.3892 / 01.2018 .9226$

\begin{abstract}
Although liver transplantation (LT) lengthens the survival time of patients with hepatocellular carcinoma (HCC), LT patients exhibit a high recurrence rate; particularly those that had advanced HCC associated with the tumor biological characteristics and long-term application of immunosuppressants. A consensus on optimal prophylaxis and treatment for recurrent HCC following LT does not currently exist. The present study retrospectively analyzed data from 36 non-University of California at San Francisco
\end{abstract}

Correspondence to: Dr Guo-Sheng Du, Department of Hepatobiliary Surgery, Organ Transplant Institute, Chinese PLA 309th Hospital, 17A Heishanhu Road, Haidian, Beijing 100091, P.R. China

E-mail: duguosheng@medmail.com.cn

*Contributed equally

Abbreviations: AFP, $\alpha$-fetoprotein; ALD, alcoholic liver disease; ALP, alkaline phosphatase; ALT, alanine aminotransferase; CT, enhanced computed tomography; DBIL, direct bilirubin; DFS, disease-free survival; FK506, tacrolimus; HBIG, human hepatitis B immunoglobulin; HBV, hepatitis B virus; HCC, hepatocellular carcinoma; HCV, hepatitis C virus; LT, liver transplantation; MRI/US, magnetic resonance imaging scan and abdominal ultrasound; mTOR, mechanistic target of rapamycin; OS, overall survival; PS-T, huaier granules; RFA, radiofrequency ablation; SRL, sirolimus; SRL+, sirolimus with huaier granules and thymalfasin; Thymalfasin, Zadaxin; TBIL, total bilirubin; TCM, traditional Chinese medicine; UCSF, University of California at San Francisco; $\gamma$-GGT, $\gamma$-glutamyl-transpeptidase

Key words: hepatocellular carcinoma, advanced, liver transplantation, sirolimus, tumor recurrence, University of California at San Francisco criteria criteria-eligible patients with advanced HCC who underwent LT, and then treated them with sirolimus (SRL)-based therapy with thymalfasin and huaier granules $(\mathrm{SRL}+, \mathrm{n}=18)$, or with tacrolimus-based therapy (controls; $n=18$ ). The SRL+ group had significantly longer recurrence times $(\mathrm{P}=0.008)$ and survival times $(\mathrm{P}<0.0001)(\mathrm{OS}, 1$-year: 100\%, 3-year: $94.4 \%$, 5-year: $77.8 \%$; DFS, 1-year: $88.9 \%$, 3-year: $55.6 \%$, 5-year: $50.0 \%)$. Furthermore, compared with pre-LT values and the control group, the SRL+ group had significantly lower serum $\alpha$-fetoprotein (AFP) levels (both $\mathrm{P}<0.0001)$ and percentage of Forkhead box P3 (FoxP3) Treg lymphocytes $(\mathrm{P}<0.001)$ during the first year. In the SRL+ group, $\mathrm{FoxP}^{+} /$cluster of differentiation $(\mathrm{CD}) 8^{+}$Treg lymphocyte percentages decreased significantly following LT $(\mathrm{P}<0.001)$; however, $\mathrm{CD}^{+} / \mathrm{CD}^{+}$ T-cells significantly increased $(\mathrm{P}<0.001)$. Levels of serum AFP and FoxP3 ${ }^{+}$Treg cells increased when tumors relapsed, and decreased to near-normal when relapse foci were cured or stabilized. SRL+ therapy may decrease AFP and Treg levels, while increasing $\mathrm{CD} 8^{+} \mathrm{T}$ cells, indicating an associated mechanism among them. In conclusion, SRL+ therapy appears to be safe and effective in preventing $\mathrm{HCC}$ recurrence following LT with no significant adverse events, and warrants further investigation.

\section{Introduction}

Liver transplantation (LT) is currently the most viable and effective treatment option for patients with hepatocellular carcinoma (HCC) except for surgical resection, thus it may be used for patients where resection is not an option (1). Although the implementation of the Milan and University of California at San Francisco (UCSF) criteria has improved post-LT survival rates, patients who do not fit the UCSF criteria currently undergo LT worldwide $(2,3)$. LT for HCC has an associated high recurrence rate, particularly for patients with advanced disease (10-60\%), in addition, the overall survival (OS) and disease-free survival (DFS) rates for post-LT patients are also higher (LT, OS vs. DFS, 70-98\% vs. 27-35\%), compared with 
those who undergo liver resection (LR OS vs. DFS, $60-72 \%$ vs. 18-37\%) (3-7). Furthermore, no consensus on the most suitable prophylaxis and treatment for recurrent HCC following LT is currently available. Although sorafenib has demonstrated efficacy for tumor recurrence $(1,2,8,9)$, the results are unsatisfactory, with a challenging adverse event profile $(10,11)$. Sorafenib also exhibits less favorable results in Asian patients compared with European patients $(9,10)$.

FK506 is an immunosuppressor that works by inhibiting calmodulin (12). It is currently used in LT patients, and is extensively used in post-LT patients with benign liver disease. However, long-term application of FK506 increases the risk of infection and de novo tumors, and particularly contributes to tumor relapse in post-LT patients with HCC (13-15). Until the introduction of sirolimus (SRL) being used with organ transplantations, there were no replacement drugs to overcome the effects of long-term use of FK506 $(15,16)$. Accumulating evidence supports the clinical safety and efficacy of immunosuppressant selection, and replacing FK506 therapy with SRL following LT for patients with HCC $(13,15-17)$. Additionally, SRL offers a dual function, as it acts as an efficacious immunosuppressor and an antineoplastic agent $(13,18,19)$, and therefore, may effectively reduce graft rejection and reduce the high relapse risk associated with long-term application of FK506.

When combined with SRL, huaier granules (PS-T), a type of traditional Chinese medicine (TCM), have been demonstrated to inhibit tumor growth, reduce vascular endothelial growth factor expression, and improve survival rates in patients with HCC $(8,18-22)$. As an immunomodulator, thymalfasin (also known as Zadaxin) promotes maturation and differentiation of $\mathrm{T}$ cells and NK cells by affecting tumor-cell-related antigen expression and differentiation, and increasing tumor immunogenicity; it has been demonstrated to be safe for post-LT patients with HCC, with no increased risk of rejection $(23,24)$. Although monotherapy with SRL led to rather effective results, no relevant research has been published on combining SRL, PS-T and thymalfasin. Although the application of SRL and huaier, thymalfasin and huaier, or single therapy, has been studied $(16-18,20)$, their effects are limited in patients with LT, and there were fewer studies regarding patients following LT $(16,20)$. Therefore, to improve survival and decrease recurrence rates, the effectiveness of a combined therapy based on SRL, thymalfasin and PS-T for LT patients with advanced HCC was investigated. In consideration of the aforementioned background information, tumor recurrence rates following LT at the Organ Transplant Institute of the Chinese PLA 309th Hospital (Beijing, China) were retrospectively analyzed to provide a potentially efficacious treatment for these patients.

\section{Materials and methods}

Patients and groups. Clinicopathological data were collected from the China Liver Transplant Registry database regarding 36 HCC patients who did not fit the UCSF Criteria, and who had undergone LT at the Organ Transplant Institute of the Chinese PLA 309th Hospital between January 2008 and January 2014, including Model for End-Stage Liver Disease (MELD) scores, Child-Pugh scores, liver function tests [alanine aminotransferase (ALT), bilirubin (BIL), alkaline phosphatase (ALP), $\gamma$-glutamyltransferase $(\gamma$-GGT)] and serum $\alpha$-fetoprotein (AFP) levels (Table I).

Patients were divided into two groups: The group treated with SRL, Zadaxin and PS-T (SRL+; $\mathrm{n}=18 ; 17$ men and 1 woman; median age, 53.94 years); and the control group ( $\mathrm{n}=18 ; 15$ men and 3 women; median age, 49.72 years). The present study was approved by the Ethics Committee of Human Experimentation of the PLA 309th Hospital (Beijing, China). Written informed consent was obtained from all patients in accordance with the Declaration of Helsinki of the World Medical Association.

Inclusion and exclusion criteria. The inclusion criteria were as follows: i) Patients in whom liver malignancy was detected by at least an enhanced computed tomography (CT) scan or magnetic resonance imaging scan and abdominal ultrasound (MRI/US) and postoperative tissue biopsies had been pathologically confirmed to be HCC; ii) whose AFP levels were $\geq 1,000 \mathrm{ng} / \mathrm{ml}$, or maintained at $400-1,000 \mathrm{ng} / \mathrm{ml}$ for 4 weeks; iii) were 30-65 years old; iv) had no distant metastases (e.g. lung, bone); and v) had undergone LT at the Organ Transplant Institute of the Chinese PLA 309th Hospital. The following exclusion criteria were maintained: i) Patients in whom preoperative CT or MRI/US indicated current malignant liver tumors and postoperative tissue biopsies had been pathologically confirmed to be cholangiocarcinoma; ii) who were $>65$ years or $<30$ years; iii) with distant metastases (e.g. lung, bone); iv) were confirmed to have hepatitis $\mathrm{C}$ virus (HCV) infection or alcoholic liver disease (ALD); or v) had concurrent severe cardiopulmonary disease or the inability to tolerate surgery.

Intra- and post-operative examining. Preoperative demographical, clinical (MELD and Child-Pugh scores) and laboratory data were recorded for these patients. Patients underwent plain/enhanced CT scan or MRI/US within 1 week before the surgery. Pathology diagnoses were considered definitive for HCC. Pathological data were considered as the standard for tumor characteristics. Microvascular invasion and tumor differentiation were also assessed by pathology.

Blood cell tests, including blood and liver function tests, is part of the routine work-up for patients with HCC who undergo LT. The Fork head box P3 (FoxP3)+ Treg and cluster of differentiation (CD) $8^{+} \mathrm{T}$ cell percentages was calculated by flow cytometry. Tumor markers were recorded prior to and following surgery. In addition, hepatitis B virus (HBV) infection was quantitatively detected prior to LT and HBV-DNA copies were assessed for $\mathrm{HBV}^{+}$patients.

Intra- and post-operative treatment. Basiliximab $(20 \mathrm{mg})$ (Simulect; Novartis International AG, Basel, Switzerland) and methylprednisolone (40 mg) (Pfizer, Inc., New York, NY, USA) were used as pre- and intra-operative induction regimens. Small doses of methylprednisolone were given post-operatively (20 mg on day $1,10 \mathrm{mg}$ on day 2 and $5 \mathrm{mg}$ on day 3 , once a day, for the first 3 days following LT for both groups) and withdrawn 1 week following LT. In addition, an immunosuppression regimen, including tacrolimus (FK506; Astellas Ireland Co., Ltd., Dublin, Ireland), with or without 
Table I. Comparison of general data between the treatment and control groups.

\begin{tabular}{|c|c|c|c|}
\hline Characteristics & Treatment group $(n=18)$ & Control group $(n=18)$ & P-value \\
\hline Sex & & & $0.603^{\mathrm{a}}$ \\
\hline Male & 17 & 15 & \\
\hline Female & 1 & 3 & \\
\hline Age (years) & $53.94 \pm 7.40$ & $49.72 \pm 7.00$ & $0.087^{\mathrm{a}}$ \\
\hline MELD & & & $0.533^{\mathrm{c}}$ \\
\hline $0-10$ & 12 & 10 & \\
\hline $10-20$ & 6 & 7 & \\
\hline$>20$ & 0 & 1 & \\
\hline Child-Pugh & & & $0.531^{\mathrm{c}}$ \\
\hline$A(5-6)$ & 11 & 9 & \\
\hline B (7-9) & 7 & 8 & \\
\hline$C(\geq 10)$ & 0 & 1 & \\
\hline AFP preoperative (ng/ml) & & & $0.489^{\mathrm{b}}$ \\
\hline $400-1,000$ & 8 & 5 & \\
\hline$>1,000$ & 10 & 13 & \\
\hline $\mathrm{HBV}$ & & & $0.614^{\mathrm{b}}$ \\
\hline Positive & 16 & 17 & \\
\hline Negative & 2 & 1 & \\
\hline ALT (ng/ml) & & & $0.738^{\mathrm{b}}$ \\
\hline$<40$ & 9 & 7 & \\
\hline$\geq 40$ & 9 & 11 & \\
\hline Bilirubin (ng/ml) & & & $0.519^{\mathrm{b}}$ \\
\hline$<34$ & 8 & 6 & \\
\hline$\geq 34$ & 10 & 12 & \\
\hline $\mathrm{Cr}(\mathrm{ng} / \mathrm{ml})$ & & & $0.282^{\mathrm{b}}$ \\
\hline$<90$ & 15 & 12 & \\
\hline$\geq 90$ & 3 & 6 & \\
\hline White protein $(\mathrm{g} / \mathrm{l})$ & & & $0.176^{\mathrm{b}}$ \\
\hline$\leq 35$ & 8 & 13 & \\
\hline$>35$ & 10 & 5 & \\
\hline
\end{tabular}

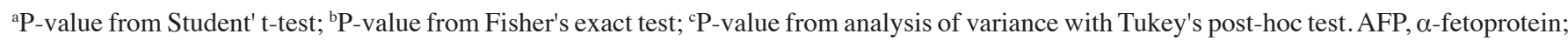
ALT, alanine transaminase; Cr, creatinine; MELD, Model for End-Stage Liver Disease; HBV, hepatitis B virus.

mycophenolate mofetil (Roche Applied Science, Madison, WI, USA), was initially administered based according to individual differences; FK506 was gradually withdrawn and completely replaced with sirolimus (Wyeth Pharmaceuticals Co. Ltd., Philadelphia, PA, USA) a month after LT. Patients were then switched to SRL combined with Zadaxin and PS-T (SRL+) following stabilization.

The thymalfasin (Zadaxin) (SciClone Pharmaceuticals, Inc., Foster City, CA, USA) was administrated as a 16-mg subcutaneous injection/day for 10 days, and then twice a week; the PS-T (Qidong Gaitianli Pharmaceutical Co., Ltd., Qidong, China) was administrated orally thrice a day (20 g/day) for $\geq 5$ year later.

For the $\mathrm{HBV}^{+}$patients, anti-viral drugs, including lamivudine or entecavir, and injection of hepatitis B immune globulin were regularly administered following LT to prevent hepatitis $B$ recurrence.
Of the 36 patients whose advanced HCC was treated with LT, 18 were treated with SRL+ and 18 were treated with an FK506-based regimen.

Follow up. The patients underwent follow-up procedures immediately following LT. All patients were required to be evaluated monthly during for the first 12 months post-LT, (including plain/enhanced CT scans, abdominal ultrasound, FoxP3 ${ }^{+}$Treg and $\mathrm{CD}^{+} \mathrm{T}$ cell percentages and serum AFP levels), then every 3 months during the second year, then every 3-6 months or when necessary in the following years. For this study, each follow-up session also included physical examinations, and the collection of data regarding rejection, survival time, tumor recurrence sites, routine blood and liver function tests, SRL concentration and HBV surface antibody titers.

If patients exhibited suspicious liver, lung or brain lesions, as indicated by imaging and blood testing, follow-up intervals 
were reduced to once a month. The date of tumor recurrence was recorded as the time the Fox $3^{+}$Treg level began to elevate, once tumor recurrence was verified. Except for the bone metastasis and progression of growth, patients with other recurrent lesions were treated by $\gamma$-radiation therapy or radiofrequency ablation (RFA)/resection (lung), RFA or transcatheter hepatic arterial chemo-embolization (liver) or resection (brain). Adverse events were also evaluated at each visit for the SRL+ group. The follow-up deadline was July 31, 2017.

Flow cytometric analysis. Analysis of the Foxp3 $3^{+}$Treg, $\mathrm{CD}^{+} \mathrm{CD}^{+}$, and $\mathrm{CD}^{+} \mathrm{CD}^{+} \mathrm{T}$ cell populations was performed for patients prior to and following LT at different time points. The analysis of Foxp $3^{+}$Treg was performed using anti-CD4, anti-CD25 and anti-FoxP3 antibodies (pre-diluted and contained in the Multitest IMK kit from eBioscience; Thermo Fisher Scientific, Inc., Waltham, MA, USA), and the analysis of the lymphocyte subpopulation was performed using the Multitest IMK kit (BD Biosciences, USA) according to the manufacturer's protocol. All the samples were detected and analyzed with BD FACSCanto clinical software (BD Biosciences). The specific testing steps as follows:

FoxP3 ${ }^{+}$Treg detection was conducted with the following procedure: i) A total of $4 \mu \mathrm{l}$ anti-CD 4 and $4 \mu 1$ anti-CD25 antibodies were added and incubated for $15 \mathrm{~min}$ at room temperature, avoiding light; ii) $1 \mathrm{ml}$ mixture containing Fixation/permeabilization concentrate $(250 \mu \mathrm{l})$ and Fixation/permeabilization diluent $(750 \mu \mathrm{l})$ (contained in the Foxp3/Transcription Factor Staining Buffer Set kit; catalog no. 00-5523-00; eBioscience; Thermo Fisher Scientific, Inc.) were mixed in a 1:3 ratio and added and incubated for $30 \mathrm{~min}$ at room temperature according to the manufacturer's protocol, avoiding light; iii) $1 \mathrm{ml} 1 \mathrm{X}$ permeabilization buffer (eBioscience; Thermo Fisher Scientific, Inc.) was added and incubated for $20 \mathrm{~min}$ at room temperature, in the dark iv) then, centrifugation was performed at $435 \mathrm{x} \mathrm{g} / \mathrm{min}$ for $5 \mathrm{~min}$ at room temperature and the supernatant was removed; v) $2 \mu 1$ anti-FoxP3 antibodies were added and incubated for $60 \mathrm{~min}$ at room temperature, avoiding light; vi) $1 \mathrm{ml} 1 \mathrm{X}$ PBS was added and centrifuged at $435 \mathrm{x} \mathrm{g} / \mathrm{min}$ for $5 \mathrm{~min}$ at room temperature; and vii), the supernatant was removed and detection was conducted with a Flow Cytometer. CD3/CD4/CD8 T cell detection was then conducted with the followed procedure: i) A total of $5 \mu \mathrm{l} \mathrm{BD}$ multitest CD3/CD8/CD45/CD4 antibodies and $5 \mu \mathrm{l} \mathrm{BD}$ multitest CD3/CD16+CD56/CD45/CD19 antibodies (BD Biosciences) were added and incubated for $15 \mathrm{~min}$ at room temperature, avoiding light; ii) $1 \mathrm{ml} 1 \mathrm{X}$ Lysing solution (BD Biosciences) was added and incubated for $30 \mathrm{~min}$ at room temperature, avoiding light; iii) then, centrifugation was performed at $435 \mathrm{x} \mathrm{g} / \mathrm{min}$ for $5 \mathrm{~min}$ at room temperature and the supernatant was removed; iv) $1 \mathrm{ml} 1 \mathrm{X}$ PBS was added and centrifuged at $435 \mathrm{x} \mathrm{g} / \mathrm{min}$ for $5 \mathrm{~min}$ at room temperature; v) the supernatant was removed detection was conducted with a Flow Cytometer.

Statistical analysis. Statistical analysis was performed using SPSS 13.0 software (SPSS, Inc., Chicago, IL, USA). Data are presented as the mean \pm standard deviation. The two groups were compared using the unpaired Student's t-test, Fisher's exact test or the Chi-square test. For multiple group comparisons using a two-way analysis of variance followed by Tukey's post hoc test. For non-normal distribution data the Wilcoxon rank-sum test was used. Patient and graft survival were calculated using Kaplan-Meier curves and the log-rank test. $\mathrm{P}<0.05$ was considered to indicate a statistically significant difference.

\section{Results}

Patient characteristics. Following the exclusion of patients with HCV infection or ALD, or who met the UCSF criteria, 36 patients were included in the present study. Among these patients, $91.7 \%(33 / 36)$ were $\mathrm{HBV}^{+}$, with $44.5 \%(16 / 36)$ in the treatment group and $47.2 \%(17 / 36)$ in the control group. The SRL+ and control groups did not significantly differ in sex or age, or in baseline MELD or Child-Pugh scores, preoperative AFP or liver function (Table I).

All the patients had pathologically diagnosed HCC following their LT, based on their liver specimens. The tumor-associated indices [tumor type (single or multiple), size, vascular invasion and pathological pattern] of the two groups are presented in Table II. All patients underwent LT for advanced liver cancer at the Organ Transplant Institute of the Chinese PLA 309th Hospital and were discharged following recovery. Patients were followed up directly following their surgeries; none dropped out during the follow-up time. Median follow-up time for the SRL+ group was 60 months (range, 47-84 months) excluding 1 case of mortality at 23 months; whereas for the control group, the median period was 15.5 months (range, 9-24 months) until the mortality of all patients.

Liver function and rejection. The indices ALT, TBIL, DBIL, ALP and $\gamma$-GGT are used to evaluate liver function and rejection. Whether preoperative liver function was abnormal or not, all the aforementioned indices typically decrease to normal levels postoperatively and remain in near-normal ranges unless rejection occurs (Table III). The SRL+ group did not vary from this pattern. The two groups did not significantly differ in rejection rates (SRL+, 2/18; controls, $4 / 18$; $\mathrm{P}>0.05)$. All the rejection cases were treated following adjustment of immunosuppressant dosages and glucocorticoid pulse therapy. No evident rejection reactions were caused by Zadaxin or SRL-based therapy throughout the follow-up period.

Imaging detection. All patients underwent regular ultrasonography and CT scans (lung and abdomen), no remarkable changes were observed in the bloodstream, bile duct or solid tissue of the liver and lung, except in cases of recurrence. Among the recurrent patients, compared with the control group, the metastasis and relapse foci in lung were significantly reduced in the SRL+ group (Fig. 1). In addition, no metastasis and relapse liver foci were detected for the lung relapse patients of the SRL+ group (Fig. 2).

AFP levels pre- and post- LT. The positive rate of liver cancer-specificity tumor marker, AFP, is $>70 \%$ among patients with liver cancer. Preoperative AFP levels of all 36 patients were positive, and did not significantly differ between the SRL+ and control groups (Fig. 3A), but did significantly differ 
Table II. Comparison of clinicopathological characteristics of two groups $(n=36)$.

\begin{tabular}{lcc}
\hline Variables & Combined group $(\mathrm{n}=18)$ & Control group $(\mathrm{n}=18)$ \\
\hline Tumor size, $\mathrm{mm}(\mathrm{SD})^{\mathrm{c}}$ & $92(38)$ & $72(36)$ \\
Tumor type, $\mathrm{n}(\%)$ & & $10(56)$ \\
Single & $4(22)$ & $8(44)$ \\
Multiple & $14(78)$ & $0.057^{\mathrm{a}}$ \\
Tumor pathology, $\mathrm{n}(\%)$ & & $7(39)$ \\
HCC & $9(50)$ & $11(61)$ \\
HCC with cirrhosis & $9(50)$ & $9(50)$ \\
Macroscopic vascular invasion, $\mathrm{n}(\%)$ & $8(44)$ & $0.738^{\mathrm{b}}$ \\
\hline
\end{tabular}

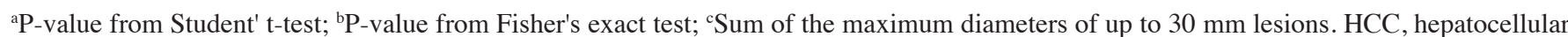
carcinoma; SD, standard deviation.

Table III. Comparison of liver function in different time points following sirolimus-based therapy.

\begin{tabular}{|c|c|c|c|c|c|}
\hline Variables & 1 year & 2 years & 3 years & $\mathrm{F}$ & P-value \\
\hline $\operatorname{ALT}(0-40 \mathrm{U} / \mathrm{l})$ & $15.97 \pm 6.79$ & $14.74 \pm 9.69$ & $14.62 \pm 5.35$ & 0.116 & 0.891 \\
\hline TBIL $(0-21 \mu \mathrm{mol} / \mathrm{l})$ & $13.84 \pm 6.48$ & $13.91 \pm 3.96$ & $16.58 \pm 2.67$ & 0.541 & 0.587 \\
\hline DBIL (0-6.8 $\mu \mathrm{mol} / \mathrm{l})$ & $3.18 \pm 1.89$ & $4.20 \pm 2.18$ & $4.06 \pm 1.49$ & 1.077 & 0.353 \\
\hline ALP (40-150 U/1) & $87.57 \pm 32.66$ & $88.18 \pm 23.99$ & $110.8 \pm 9.83$ & 1.442 & 0.252 \\
\hline$\gamma$-GGT (8-58 U/l) & $42.79 \pm 23.79$ & $35.63 \pm 14.92$ & $50.40 \pm 14.02$ & 0.988 & 0.384 \\
\hline
\end{tabular}

All P-values were calculated using the Wilcoxon rank sum test. ALT, alanine transaminase; TBIL, total bilirubin; DBIL, direct bilirubin; ALP, alkaline phosphatase; $\gamma$-GGT, $\gamma$-glutamyl transpeptidase.

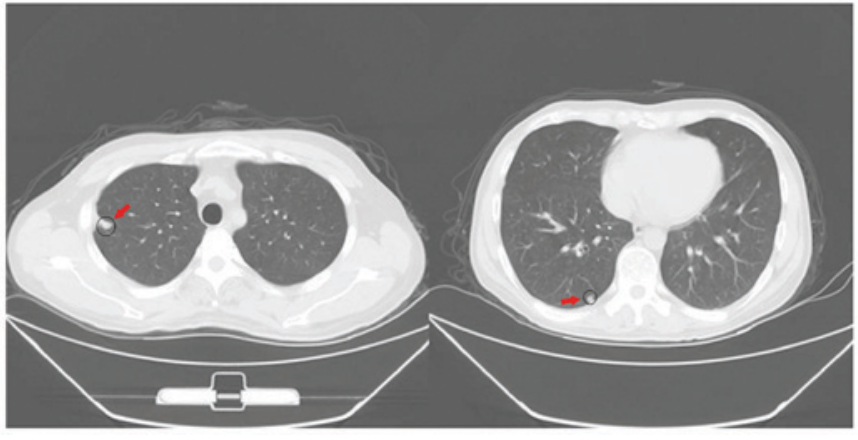

Figure 1. Lung metastases and relapse foci (red arrows) in relapsed treatment-group patients.

at 1 year post-LT (Fig. 3A). Post-LT AFP titers in the SRL+ group dropped and were maintained at normal levels for a long time, although for the recurrent patients, the AFP titers may remain at low levels $(<400 \mathrm{ng} / \mathrm{ml})$ or decline to normal for some time (Fig. 3B and C). Furthermore, the tumor recurrence time of the SRL+ group was significantly longer, compared with the control group (Fig. 3D).

T-cell-associated indices. In addition to AFP, Tregs, particularly Foxp $3^{+}$Tregs, are negatively correlated with prognosis in patients with HCC, and have been used as an independent predictor of tumor recurrence (20). The percentage of Treg

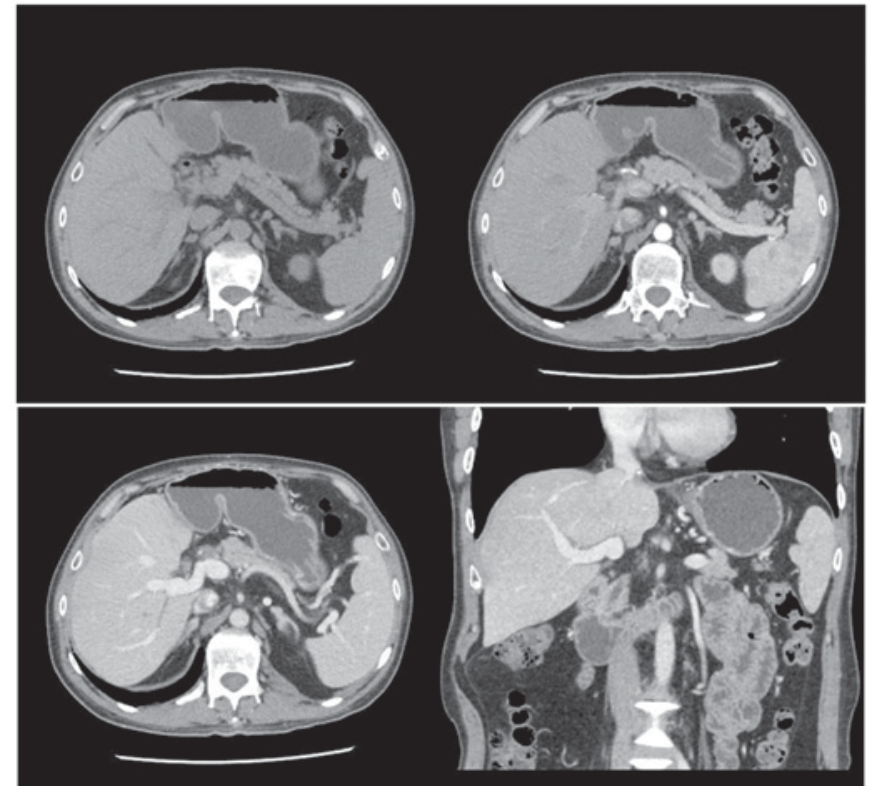

Figure 2. Liver computed tomography scan results for the lung-relapse patients in the treatment group.

(Treg\%) and Foxp3 $3^{+}$Treg (Foxp3 ${ }^{+}$Treg\%) among lymphocytes, and the ratios for $\mathrm{CD}^{+} \mathrm{T} / \mathrm{CD}^{+} \mathrm{T}$ cells and for FoxP3 ${ }^{+}$ Treg/CD $8^{+} \mathrm{T}$ cells at 1,3 and 5 years after LT were used to 
A

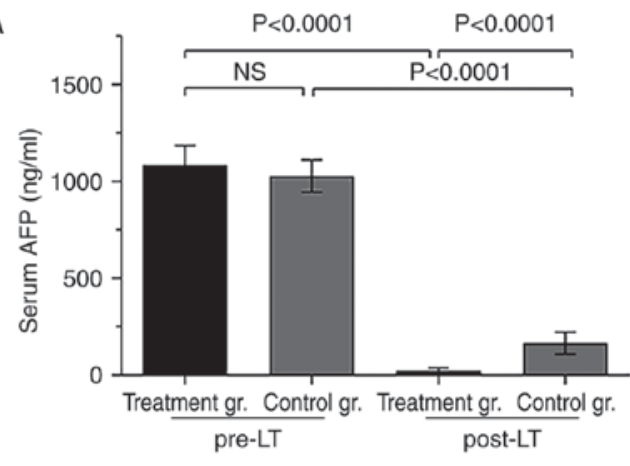

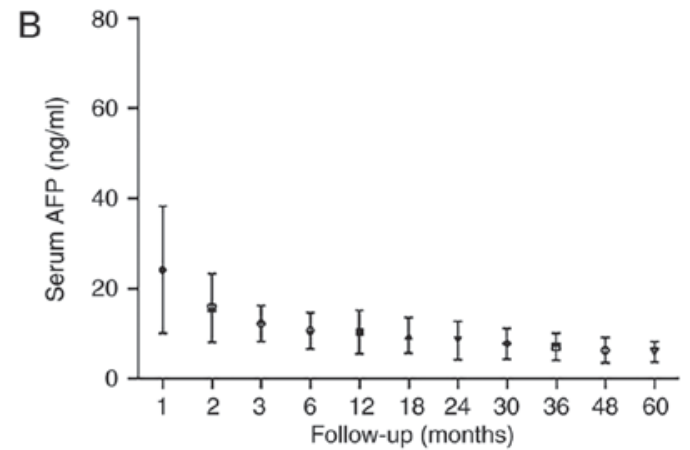

C

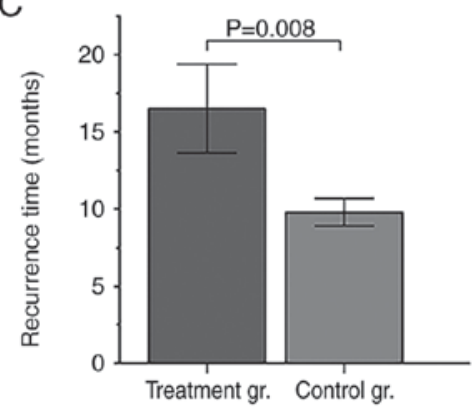

D 400

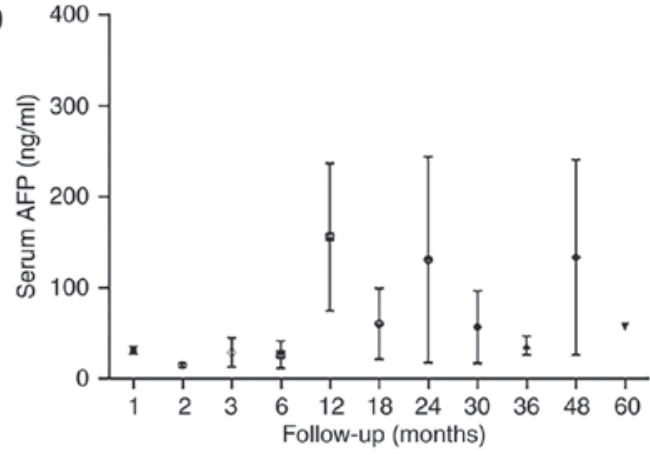

Figure 3. Serum AFP and recurrence times for the treated (SRL+) and control groups, prior to and following LT. (A) Serum AFP changes in SRL+ and control groups at pre-LT and post-LT. (B) Serum AFP changes of non-recurrent cases following SRL+ therapy after LT. (C) Tumor recurrence times in SRL+ and control groups. (D) Serum AFP changes for recurrent cases following SRL+ therapy. Treatment gr., SRL+ group; control gr., control group; AFP, $\alpha$-fetoprotein; SRL, sirolimus; SRL+, SRL combined with Zadaxin and huaier granules; LT, liver transplantation; NS, not significant.
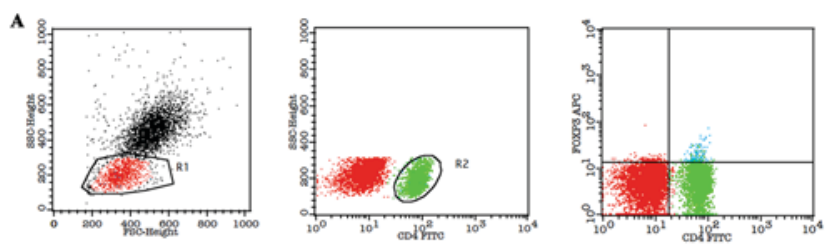

(1)
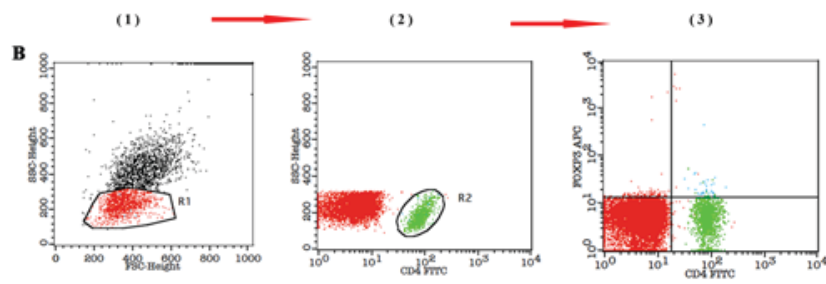

(1)

(2)

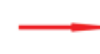

(3)
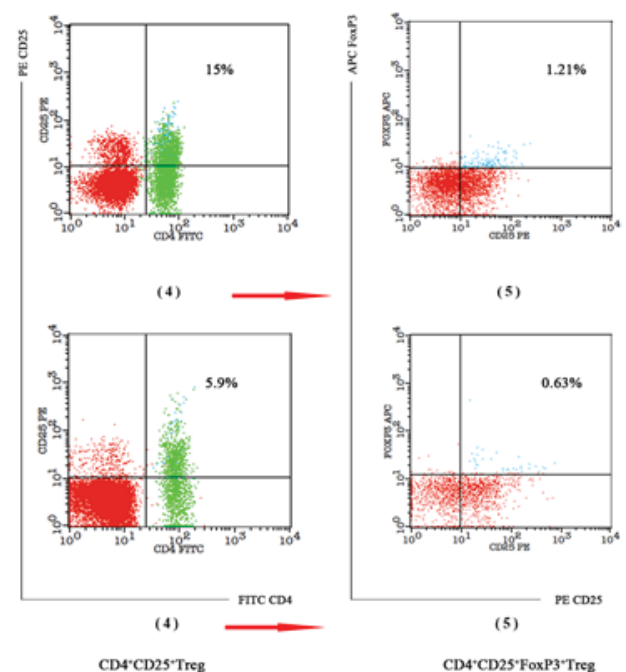

$(5$

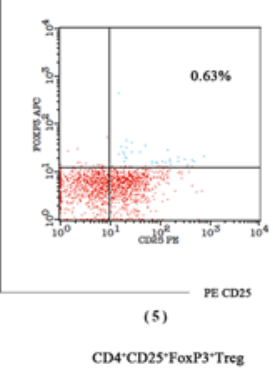

Figure 4. Changes in Treg and Foxp3+ Treg percentages, pre-LT and post-LT. (A) Pre-LT Treg and Foxp3 ${ }^{+}$Treg percentages of lymphocytes (Treg, $15 \%$; Foxp3 ${ }^{+}$ Treg, 1.21\%). (B) Percentages of Treg and Foxp3 ${ }^{+}$Treg in lymphocytes at 1 year post-LT (Treg, 5.9\%; Foxp3 ${ }^{+}$Treg, 0.63\%). FoxP3, Forkhead box P3; LT, liver transplantation; FITC, fluorescein isothiocyanate; PE, phycoerythrin; CD, cluster of differentiation; SSC, side scatter height; FSC, forward scatter height; APC, allophycocyanin.

evaluate anti-cancer effects and predict survival benefits. Preoperative Treg\%, Foxp3 $3^{+}$Treg\% and FoxP $3^{+}$Treg/CD ${ }^{+}$ $\mathrm{T}$ cell ratios were significantly higher at 1,3 and 5 years compared with immediately following treatment (all $\mathrm{P}<0.05$; Fig. 4; Table IV). Furthermore, the association of CD8 ${ }^{+} \mathrm{T} / \mathrm{CD}^{+} \mathrm{T}$ preoperatively and postoperatively at different time points was indirect (Table IV). Compared with the preoperative result, as the level of Foxp3 $3^{+}$Treg\% gradually reduced, the level of $\mathrm{CD} 8^{+} \mathrm{T} / \mathrm{CD}^{+}{ }^{+} \mathrm{T}$ increased gradually. This indicated a direct association between the reduced level of FoxP3 ${ }^{+}$Treg and the reduced $\mathrm{T}$ cell inhibition.

Higher FoxP3 titers in relapsed patients gradually decreased to levels observed in non-relapsed patients following $\gamma$-radiation and SRL-based therapy (Fig. 5). 
Table IV. Changes in T cell-associated tumor recurrence index in treatment group at different time points.

\begin{tabular}{|c|c|c|c|c|c|c|c|}
\hline Variables & $\mathrm{n}=36$ & Foxp $3^{+}$Treg $\%$ a & F-value & $\mathrm{CD}^{+} \mathrm{T} / \mathrm{CD}^{+} \mathrm{T}^{\mathrm{a}}$ & F-value & $\mathrm{FoxP}^{+}{ }^{+}$Treg $/ \mathrm{CD}^{+} \mathrm{T}^{\mathrm{a}}$ & F-value \\
\hline Pre-LT & 18 & $1.23 \pm 0.35$ & 62.11 & $0.31 \pm 0.07$ & 9.16 & $0.07 \pm 0.02$ & 83.89 \\
\hline Post-LT (1 year) & 18 & $0.38 \pm 0.16$ & & $0.47 \pm 0.15$ & & $0.02 \pm 0.01$ & \\
\hline Post-LT (3 year) & 17 & $0.36 \pm 0.14$ & & $0.43 \pm 0.08$ & & $0.012 \pm 0.005$ & \\
\hline Post-LT (5 year) & 10 & $0.29 \pm 0.15$ & & $0.47 \pm 0.06$ & & $0.014 \pm 0.004$ & \\
\hline
\end{tabular}

${ }^{\mathrm{a}} \mathrm{P}<0.01$, compared with pre-LT level, the P-values were calculated using the Wilcoxon rank sum test. Pre-LT, pre-liver transplant; post-LT, post-liver transplant; FoxP3, Forkhead box P3; CD, cluster of differentiation.

Tumor recurrence and treatment. After 12 months, 4 patients in the SRL+ group had suffered tumor recurrences; of whom 2 had pulmonary metastases, but no liver recurrence or other metastases. Another patient was identified to have pulmonary metastases at 18 months after LT. The patient obtained survival benefits with tumor silencing without progression following $\gamma$-radiation therapy; unfortunately, the patient was diagnosed with brain metastases 50 months after LT, but survived well after resection of the metastases (Table V).

Notably, although 1 patient with multiple recurrences succumbed from tumor complications, the others achieved a good quality of life and survived well after $\gamma$-radiation therapy when the data were collected (Table V). The higher AFP and FoxP3 levels in relapsed patients also declined to near-normal levels, similar to those of non-relapsed patients (Fig. 5). By contrast, 10 control patients (10/18) had tumor recurrences during the first 3 to 12 months post-LT, and the other 8 had recurrences at 12 to 15 months; even following positive $\gamma$-radiation therapy and chemotherapy, all the patients in the control group succumbed within 24 months from multiple tumor metastases and resulting complications.

Survival benefits. In addition to the recurrent patients, other patients of the SRL+ group survived well past-transplantation till the deadline of data collection. The respective 1-year OS and DFS rates were 100 and $88.9 \%$ for the SRL+ group, and 77.8 and $22.2 \%$ in the control group. Whereas none of the control patients lived $>2$ years post-LT, the OS rates at 3 and 5 years 94.5 and $77.8 \%$, respectively in the SRL+ group. In addition, the DFS rates at 3 and 5 years were 55.6 and $50.0 \%$ in the SRL+ group, respectively. The survival curves demonstrated that the SRL+ group had a significantly longer survival time $(\mathrm{P}<0.001)$ compared with the control group, although the groups did not significantly differ in baseline values that influence patient survival, including age and rejection rate (Fig. 6).

\section{Discussion}

LT is the currently only effective treatment for advanced liver cancer, and may improve patient prognosis and quality of life. However, patients who undergo LT have a high risk of tumor recurrence due to long-term use of FK506; whereby subsequent administration of immunosuppressive drugs may accelerate the risk of mortality $(1-3,25)$. Although current treatments for post-LT recurrence in patients with advanced
HCC has limited efficacy (2,5,8-11), the recent 'SiLIVER' study demonstrated that SRL may act as an efficacious immunosuppressive drug and an antineoplastic agent for LT patients, with favorable clinical safety and efficacy comparable to that of FK506 (13). This trial indicated that switching to SRL from FK506 following LT significantly improved the survival rates for patients with $\mathrm{HCC}$ in the first 3 to 5 years, particularly among low-risk patients, including those who meet the Milan criterion (13). It also provided the first high-level evidence for selecting SRL-based immunosuppression in LT recipients with HCC (13). Similarly, this retrospective analysis of the combined application of SRL, Zadaxin and PS-T has revealed promising results with long-term survival and low relapse rates.

Despite the high recurrence rate of HCC following LT (10-60\%), OS and DFS rates are hopeful, at 70-98\% and $60-72 \%$, respectively, following LT $(3-4,7,13,16,26)$. The present study demonstrated that the SRL-based therapy improved post0LT survival and decreased recurrence rates compared with the control group (OS, 1-year: 100\%, 3-year: 94.4\%, 5-year: 77.8\%; DFS, 1-year: $88.9 \%$, 3-year: $55.6 \%$, 5-year: $50.0 \%$ ). Furthermore, the tumor recurrence rate in the present study was 11.1 and $22.2 \%$ for 1 and 3 years, respectively, and there were no new relapse cases beyond 3 years.

Although single applications of SRL are reportedly effective in preventing post-transplantation recurrence $(13,16)$, they were only $10-30 \%$ effective for patients who met the Milan criteria $(8,13)$. Excessive SRL doses also have serious side effects, including myelosuppression, thrombocytopenia and interstitial pneumonia, which are not observed with FK506 treatment $(8,18)$. In the present study, it was indicated that the therapeutic concentration of SRL does not rely on drug dosage, but primarily on liver and renal function, rejection status and anti-tumor effect. Therefore, it is suggested that serum SRL levels should be maintained at $\leq 10 \mathrm{ng} / \mathrm{ml}$ to avoid severe adverse reactions. Furthermore, as FK506 and SRL doses are collocated regularly, FK506 should be gradually withdrawn, starting during the second month after LT.

Research on anticancer TCM for solid malignant tumors is increasing and yielding promising findings. Trametes robiniophila Murr. has been used for $>1,600$ years in TCM practice as a medicinal fungus for the treatment of inflammation and cancer in China $(22,27)$, and the TCM PS-T has been used as an adjuvant drug for chemoradiotherapy, with 
Table V. Treatment and survival status of the patients with tumor recurrence.

\begin{tabular}{lcllll}
\hline Case & $\begin{array}{c}\text { Recurrence } \\
\text { time/months }\end{array}$ & $\begin{array}{l}\text { Recurrence } \\
\text { site/number }\end{array}$ & $\begin{array}{c}\text { Treatment } \\
\text { method }\end{array}$ & \multicolumn{1}{c}{$\begin{array}{c}\text { Therapeutic } \\
\text { effect }\end{array}$} & Prognosis \\
\hline 1 & 12 & Lung/single & $\gamma$-radiation & Cured & Survived \\
3 & 12 & Lung/two & $\gamma$-radiation & Remission & Succumbed at 23 months after LT \\
& 18 & Lung/two & $\gamma$-radiation & Tumor silence, no progress & Survived \\
& 50 & Brain/single & Surgical resection & Cured & Survived \\
\hline
\end{tabular}

LT, liver transplantation.

A

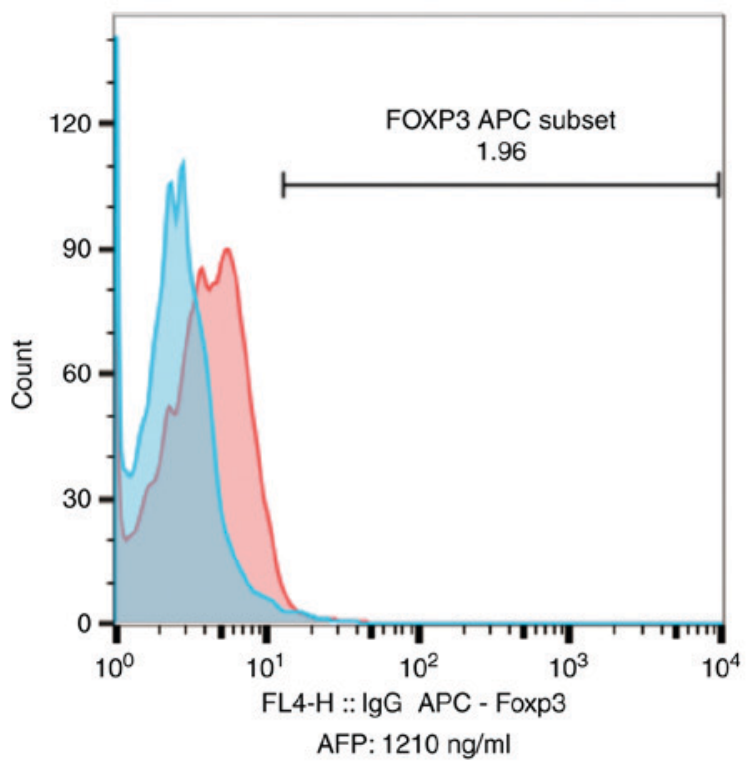

B

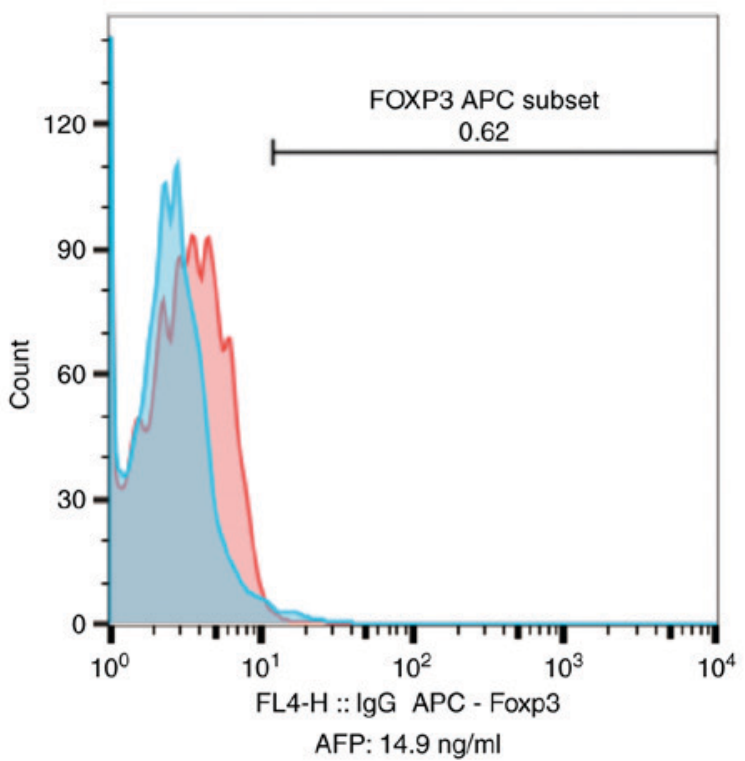

Figure 5. Changes in Foxp3 ${ }^{+}$and Treg percentages of lymphocytes, and serum AFP levels in recurrent patients (A) prior to and (B) following therapy. AFP, $\alpha$-fetoprotein; FoxP3, Forkhead box P3; APC, allophycocyanin.

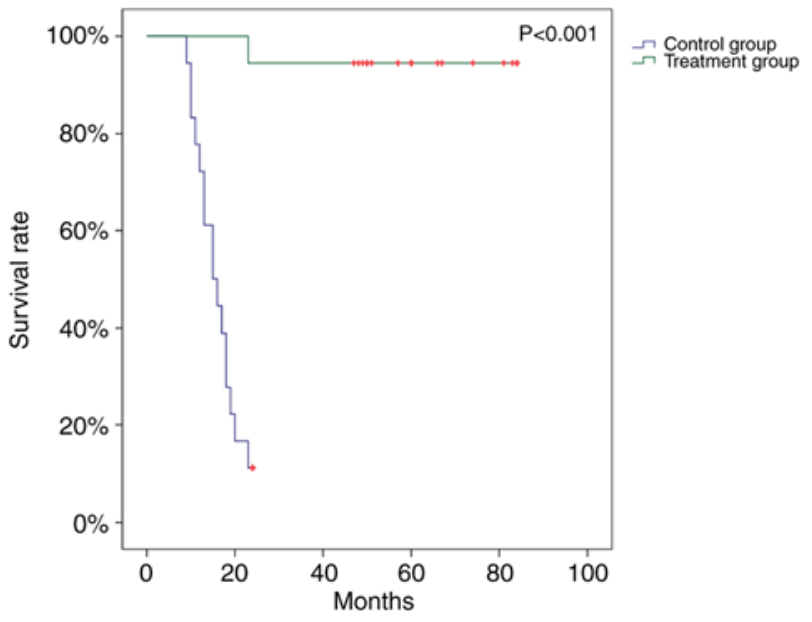

Figure 6. Survival rates of the patients with hepatocellular carcinoma following liver transplantation in the treated and control groups $(\mathrm{P}<0.001)$.

good clinical efficacy against liver, lung, gastric and breast cancer (22,27-29). PS-T has been revealed to be a multitarget drug with proteoglycans that improve immune functions, particularly those of $\mathrm{T}$ effector cells and natural killer cells, and kill tumor cells $(29,30)$. Thus, it was considered that PS-T alone or in combination with other drugs may improve the quality of life and prolong the survival time of patients with HCC treated with resection and LT.

In vivo research on PS-T in combination with SRL has confirmed that combined therapy inhibits the tumorigenesis through activation of mechanistic target of rapamycin (mTOR) signaling by PS-T, thus increasing the sensitivity of cells more effectively compared with PS-T or SRL alone (29). In the present study, including PS-T in post-LT treatment (taken continuously, $20 \mathrm{~g}$ orally, 3/day, started as soon as possible following LT) exhibited survival benefits compared with FK506-based therapy. Increasing numbers of studies on the treatment of patients with HCC with SRL combined with PS-T post-LT have been conducted in Chinese medical centers (31-33).

The effects of the mTOR signaling pathway on cell growth, and of $\mathrm{CD}^{+} \mathrm{T}$ cells (CTLs) and FoxP3 ${ }^{+}$Tregs on antitumor activity are involved in immune tolerance induction by SRL $(34,35)$. Numerous studies have identified a negative association between high FoxP3 ${ }^{+}$Tregs levels and the prognosis 
of patients with $\mathrm{HCC}$, as well as a positive association with the risk of tumor relapse $(36,37)$. Furthermore, Zadaxin may improve cellular immune function of CTLs and affect FoxP3 ${ }^{+}$ Treg function. Certain centers have attempted to use Zadaxin in organ transplantation, and have indicated that it does not increase the risk of rejection even when it was theoretically demonstrated to be infeasible (38-40). Therefore, Zadaxin was used in this study to prevent tumor relapse. It did not increase the rejection rate or cause other adverse events. In the present study, the SRL-based therapy significantly downregulated expression of FoxP3 ${ }^{+}$Treg and increased CTL levels, to those comparable with pre-LT levels and relapse cases. To improve the cellular immune function of patients with HCC, early application of Zadaxin following LT is recommended as a subcutaneous injection of 1.6-mg dose 1/day for 10 days, followed by $2 /$ week, with long-term maintenance.

Application of immunosuppressant produces a systemically inhibitory effect, which may affect the immune system, not just the $\mathrm{CD}^{+}{ }^{+} \mathrm{T}$ lymphocytes (40). Foxp3 expression is the highest in $\mathrm{CD}^{+} \mathrm{T}$ lymphocytes, but is also partially expressed in $\mathrm{CD} 8^{+} \mathrm{T}$, NK lymphocytes and other lymphocytes $(38,41)$. Tumor immunity for HCC and allograft tolerance for LT are associated with FoxP $3^{+}$Tregs $(34,42)$. However, allograft tolerance induction requires high levels of FoxP3 ${ }^{+}$Tregs (35). Thus, FoxP3 ${ }^{+}$Tregs may exhibit contradictory roles in immune tolerance induction $(43,44)$ and tumor recurrence prevention in patients with HCC following LT $(34,45)$. Additionally, FoxP3+ Tregs directly and indirectly interact with $\mathrm{CD}^{+}$and $\mathrm{CD}^{+} \mathrm{T}$ cells $(43,44,46)$. CTLs are important effector cells in antitumor reactions and graft rejection. During the present study, it was observed that SRL-based therapy decreased FoxP3 ${ }^{+}$Tregs among lymphocytes and the FoxP3 ${ }^{+}$Treg/CD8 ${ }^{+} \mathrm{T}$ cell ratio, and increased the $\mathrm{CD}^{+} / \mathrm{CD}^{+} \mathrm{T}$-cell ratio. It was also demonstrated that these cell levels may supply sufficient suppression strength and immune ability for surveillance of the tumor cell relapse due to the lower and delayed tumor recurrence. SRL-based therapy may also decrease serum AFP, which is consistent with the Treg trends. We hypothesize that regulation of Tregs through mTOR signaling may be the mechanism through which SRL-based therapy prevents recurrence.

In the present study, the primary immunosuppressant used post-transplantation was SRL. When SRL was combined with Zadaxin and PS-T, the SRL-based therapy significantly prolonged OS and DFS time and decreased post-LT tumor recurrence rate, whereby $22 \%(4 / 18)$ of the SRL+ group suffered recurrence, compared with $100 \%(18 / 18)$ in the control group. It was also suggested that monitoring post-LT changes in FoxP $3^{+}$ Treg and $\mathrm{CD}^{+} \mathrm{T}$ cell levels along with serum AFP levels is a reliable predictive index of tumor recurrence and long-term survival in patients whose advanced HCC is treated with LT.

This clinical experiment was limited by its small study cohort, short observation time and single-institution design. Larger, multi-center studies are required to verify the conclusions of the current study. In addition, animal experiments are required to investigate the underlying mechanism of the combined treatment, and to determine the relevant molecular and cellular changes.

The present study has demonstrated the safety and efficacy of Zadaxin use in patients with HCC following LT. In conclusion, early application of SRL-based therapy following LT in advanced HCC may improve quality of life and delay tumor recurrence without increasing the rejection rate. Therefore, this clinical study performed at the Organ Transplant Institute of the Chinese PLA 309th Hospital suggests a novel regimen to prevent tumor recurrence in patients treated with LT for advanced $\mathrm{HCC}$.

\section{Acknowledgements}

Not applicable.

\section{Funding}

The present study was supported by the Natural Science Foundation of China (grant no. 81771717).

\section{Availability of data and materials}

The datasets used and/or analyzed during the current study are available from the corresponding author on reasonable request.

\section{Authors' contributions}

LZ, LCP, YGZ and GSD designed the study. LZ, LCP, YGZ, XQF, XZS, LLS and SZY collected the data. LZ, JYS, WC, DHZ and ZDZ analyzed the data and LZ, LCP, DHZ and LLS drew the diagrams. LZ, LCP and GSD drafted the manuscript. GSD, DHZ and ZJL made critical revisions and approved the paper.

\section{Ethics approval and consent to participate}

The present study was approved by the Ethics Committee of Human Experimentation of the Chinese PLA 309th Hospital (Beijing, China). Written informed consent was obtained from all patients in accordance with the Declaration of Helsinki of the World Medical Association.

\section{Consent for publication}

Not applicable.

\section{Competing interests}

The authors declare that they have no competing interests.

\section{References}

1. Yan J, Tan C, Gu F, Jiang J, Xu M, Huang X, Dai Z, Wang Z, Fan J and Zhou J: Sorafenib delays recurrence and metastasis after liver transplantation in a rat model of hepatocellular carcinoma with high expression of phosphorylated extracellular signal-regulated kinase. Liver Transpl 19: 507-520, 2013

2. Roh YN, David Kwon CH, Song S, Shin M, Man Kim J, Kim S, Joh JW and Lee SK: The prognosis and treatment outcomes of patients with recurrent hepatocellular carcinoma after liver transplantation. Clin Transplant 28: 141-148, 2014.

3. Alsina AE: Liver transplantation for hepatocellular carcinoma Cancer Control 17: 83-86, 2010.

4. Baccarani U, Isola M, Adani GL, Benzoni E, Avellini C, Lorenzin D, Bresadola F, Uzzau A, Risaliti A, Beltrami AP, et al: Superiority of transplantation versus resection for the treatment of small hepatocellular carcinoma. Transpl Int 21: 247-254, 2008 . 
5. Kim HR, Cheon SH, Rha SY, Lee S, Han KH, Chon CY, Lee JD, Sung JS and Chung HC: Treatment of recurrent hepatocellular carcinoma after liver transplantation. Asia Pac J Clin Oncol 7: 258-269, 2011

6. Lee KK, Kim DG, Moon IS, Lee MD and Park JH: Liver transplantation versus liver resection for the treatment of hepatocellular carcinoma. J Surg Oncol 101: 47-53, 2010.

7. Shah SA, Cleary SP, Wei AC, Yang I, Taylor BR, Hemming AW, Langer B, Grant DR, Greig PD and Gallinger S: Recurrence after liver resection for hepatocellular carcinoma: Risk factors, treatment, and outcomes. Surgery 141: 330-339, 2007.

8. Gomez-Martin C, Bustamante J, Castroagudin JF, Salcedo M, Garralda E, Testillano M, Herrero I, Matilla A and Sangro B: Efficacy and safety of sorafenib in combination with mammalian target of rapamycin inhibitors for recurrent hepatocellular carcinoma after liver transplantation. Liver Transpl 18: 45-52, 2012.

9. Tan WF, Qiu ZQ, Yu Y, Ran RZ, Yi B, Lau WY, Liu C, Qiu YH, Feng FL, Wang JH, et al: Sorafenib extends the survival time of patients with multiple recurrences of hepatocellular carcinoma after liver transplantation. Acta Pharmacol Sin 31: 1643-1648, 2010.

10. Staufer K, Fischer L, Seegers B, Vettorazzi E, Nashan B and Sterneck M: High toxicity of sorafenib for recurrent hepatocellular carcinoma after liver transplantation. Transpl Int 25: 1158-1164, 2012.

11. Zavaglia C, Airoldi A, Mancuso A, Vangeli M, Viganò R, Cordone G, Gentiluomo M and Belli LS: Adverse events affect sorafenib efficacy in patients with recurrent hepatocellular carcinoma after liver transplantation: Experience at a single center and review of the literature. Eur J Gastroenterol Hepatol 25: 180-186, 2013.

12. Mueller AR, Platz KP, Bechstein WO, Schattenfroh N, Stoltenburg-Didinger G, Blumhardt G, Christe W and Neuhaus P: Neurotoxicity after orthotopic liver transplantation. A comparison between cyclosporine and FK506. Transplantation 58 155-170, 1994.

13. Geissler EK, Schnitzbauer AA, Zülke C, Lamby PE, Proneth A, Duvoux C, Burra P, Jauch KW, Rentsch M, Ganten TM, et al: Sirolimus use in liver transplant recipients with hepatocellular carcinoma: A randomized, multicenter, Open-Label phase 3 trial. Transplantation 100: 116-125, 2016.

14. Alessiani M, Cillo U, Fung JJ, Irish W, Abu-Elmagd K, Jain A, Takaya S, Van Thiel D and Starzl TE: Adverse effects of FK 506 overdosage after liver transplantation. Transplant Proc 25: 628-634, 1993.

15. Taniai N, Akimaru K, Ishikawa Y, Kanada T, Kakinuma D, Mizuguchi Y, Mamada Y, Yoshida $\mathrm{H}$ and Tajiri T: Hepatotoxicity caused by both tacrolimus and cyclosporine after living donor liver transplantation. J Nippon Med Sch 75: 187-191, 2008.

16. Zhang ZH, Li LX, Li P, Lv SC, Pan B and He Q: Sirolimus in liver transplant recipients with hepatocellular carcinoma: An updated meta-analysis. J Invest Surg: 1-10, 2018.

17. Toso C, Merani S, Bigam DL, Shapiro AM and Kneteman NM: Sirolimus-based immunosuppression is associated with increased survival after liver transplantation for hepatocellular carcinoma. Hepatology 51: 1237-1243, 2010.

18. Guba M, von Breitenbuch P, Steinbauer M, Koehl G, Flegel S, Hornung M, Bruns CJ, Zuelke C, Farkas S, Anthuber M, et al: Rapamycin inhibits primary and metastatic tumor growth by antiangiogenesis: Involvement of vascular endothelial growth factor. Nat Med 8: 128-135, 2002.

19. Shao H, Gao C, Tang H, Zhang H, Roberts LR, Hylander BL, Repasky EA, Ma WW, Qiu J, Adjei AA, et al: Dual targeting of mTORC1/C2 complexes enhances histone deacetylase inhibitor-mediated anti-tumor efficacy in primary HCC cancer in vitro and in vivo. J Hepatol 56: 176-183, 2012.

20. Li C, Wu X, Zhang H, Yang G, Hao M, Sheng S, Sun Y, Long J, $\mathrm{Hu}$ C, Sun X, et al: A Huaier polysaccharide inhibits hepatocellular carcinoma growth and metastasis. Tumour Biol 36 : 1739-1745, 2015.

21. Wang X, Zhang N, Huo Q and Yang Q: Anti-angiogenic and antitumor activities of Huaier aqueous extract. Oncol Rep 28 : $1167-1175,2012$

22. Zhang N, Kong X, Yan S, Yuan C and Yang Q: Huaier aqueous extract inhibits proliferation of breast cancer cells by inducing apoptosis. Cancer Sci 101: 2375-2383, 2010.
23. Garaci E, Pica F, Serafino A, Balestrieri E, Matteucci C, Moroni G, Sorrentino R, Zonfrillo M, Pierimarchi P and Sinibaldi-Vallebona P: Thymosin $\alpha 1$ and cancer: Action on immune effector and tumor target cells. Ann N Y Acad Sci 1269: 26-33, 2012.

24. Serafino A, Pierimarchi P, Pica F, Andreola F, Gaziano R, Moroni N, Zonfrillo M, Sinibaldi-Vallebona P and Garaci E: Thymosin $\alpha 1$ as a stimulatory agent of innate cell-mediated immune response. Ann N Y Acad Sci 1270: 13-20, 2012.

25. Alsina AE, Nakshabandi A, Makris AM and Torres EA: Liver transplantation for hepatocellular carcinoma in Puerto Ricans: Underutilization of a curative therapy. P R Health Sci J 33: 170-176, 2014.

26. Mathai AM, Kapadia MJ, Alexander J, Kernochan LE, Swanson PE and Yeh MM: Role of Foxp3-positive tumor-infiltrating lymphocytes in the histologic features and clinical outcomes of hepatocellular carcinoma. Am J Surg Pathol 36: 980-986, 2012

27. Chen L, Lu P, Lu Z and Dechun L: Anticancer effect of PS-T on the experimental hepatocellular carcinoma. Chin German J Clin Oncol 3: 55-59, 2004.

28. Wang CY, Bai XY and Wang CH: Traditional Chinese medicine: A treasured natural resource of anticancer drug research and development. Am J Chin Med 42: 543-559, 2014.

29. Hu Z, Yang A, Fan H, Wang Y, Zhao Y, Zha X, Zhang H and Tu P: Huaier aqueous extract sensitizes cells to rapamycin and cisplatin through activating mTOR signaling. J Ethnopharmacol 186: 143-150, 2016.

30. Sun Y, Sun T, Wang F, Zhang J, Li C, Chen X, Li Q and Sun S: A polysaccharide from the fungi of Huaier exhibits anti-tumor potential and immunomodulatory effects. Carbohydr Polym 92: 577-582, 2013.

31. Zhou L, Su XJ, Pan LC, Du GS, Zheng YG, Xiao L, Kong XR, Gao Y, Zhu ZD, Song JY, et al: Long-Time survival experience of sirolimus in combination with thymosin alpha -1 and huaier granule for prevention tumor-recurrence in liver transplantation recipient of advanced intrahepatic cholangiocarcinoma: A case report. Ausin J Cancer Clin 1: 1075, 2017.

32. Lei JY, Yan LN, Zhu JQ and Wang WT: Hepatocellular carcinoma patients may benefit from postoperative huaier aqueous extract after liver transplantation. Transplant Proc 47: 2920-2924, 2015.

33. Zhou L, Du GS, Pan LC, Zheng YG, Liu ZJ, Shi HD, Yang SZ, Shi XJ, Xuan M, Feng LK and Zhu ZD: Sirolimus treatment for cirrhosis or hepatocellular carcinoma patients accompanied by psoriasis after liver transplantation: A single center experience. Oncol Lett 14: 7817-7824, 2017.

34. Chen X, Du Y and Huang Z: CD4+CD25+ Treg derived from hepatocellular carcinoma mice inhibits tumor immunity. Immunol Lett 148: 83-89, 2012.

35. Lee WC, Wu TJ, Chou HS, Yu MC, Hsu PY, Hsu HY and Wang CC: The impact of CD4+CD25+ T cells in the tumor microenvironment of hepatocellular carcinoma. Surgery 151: 213-222, 2012.

36. Li F, Guo Z, Lizée G, Yu H, Wang H and Si T: Clinical prognostic value of $\mathrm{CD} 4+\mathrm{CD} 25+\mathrm{FOXP} 3+$ regulatory $\mathrm{T}$ cells in peripheral blood of Barcelona Clinic Liver Cancer (BCLC) stage B hepatocellular carcinoma patients. Clin Chem Lab Med 52: 1357-1365, 2014.

37. Ozgur HH, Ercetin AP, Eliyatkin N, Seren A, Kupelioglu A, Ortac R, Diniz G and Aktas S: Regulatory $T$ cells and their prognostic value in hepatopancreatobiliary tumours. Hepatogastroenterology 61: 1847-1851, 2014.

38. Romani L, Bistoni F, Montagnoli C, Gaziano R, Bozza S, Bonifazi P, Zelante T, Moretti S, Rasi G, Garaci E and Puccetti P: Thymosin alpha1: An endogenous regulator of inflammation, immunity, and tolerance. Ann N Y Acad Sci 1112: 326-338, 2007.

39. Ji SM, Li LS, Sun QQ, Chen JS, Sha GZ and Liu ZH: Immunoregulation of thymosin alpha 1 treatment of cytomegalovirus infection accompanied with acute respiratory distress syndrome after renal transplantation. Transplant Proc 39: 115-119, 2007.

40. Wolf E, Milazzo S, Boehm K, Zwahlen M and Horneber M: Thymic peptides for treatment of cancer patients. Cochrane Database Syst Rev: CD003993, 2011.

41. Joosten SA, van Meijgaarden KE, Savage ND, de Boer T, Triebel F, van der Wal A, de Heer E, Klein MR, Geluk A and Ottenhoff TH: Identification of a human CD8+ regulatory T cell subset that mediates suppression through the chemokine CC chemokine ligand 4. Proc Natl Acad Sci USA 104: 8029-8034, 2007. 
42. Huang Y, Liao H, Zhang Y, Yuan R, Wang F, Gao Y, Wang P and Du Z: Prognostic value of Tumor-Infiltrating FoxP3+ T cells in gastrointestinal cancers: A meta analysis. PLoS One 9: e94376, 2014.

43. Pons JA, Revillanuin B, Ramírez P, Baroja-Mazo A and Parrilla P: Development of immune tolerance in liver transplantation. Gastroenterol Hepatol 34: 155-169, 2011 (In Spanish).

44. Karczewski M, Karczewski J, Kostrzewa A, Wiktorowicz K and Glyda M: The role of foxp3+ regulatory T cells in kidney transplantation. Transpl Proc 41: 1527-1529, 2009.

45. Shang B, Liu Y, Jiang SJ and Liu Y: Prognostic value of tumor-infiltrating FoxP3+ regulatory $\mathrm{T}$ cells in cancers: A systematic review and meta-analysis. Sci Rep 5: 15179, 2015.
46. Casares N,Arribillaga L,Sarobe P,Dotor J,Lopez-Diaz deCerio A, Melero I, Prieto J, Borrás-Cuesta F and Lasarte JJ: CD4+/CD25+ regulatory cells inhibit activation of tumor-primed CD4+ T cells with IFN-gamma-dependent antiangiogenic activity, as well as long-lasting tumor immunity elicited by peptide vaccination. J Immunol 171: 5931-5939, 2003.

cc) (i) (3) This work is licensed under a Creative Commons Attribution-NonCommercial-NoDerivatives 4.0 International (CC BY-NC-ND 4.0) License. 